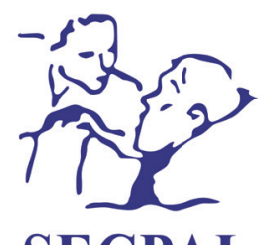

Medicina Paliativa

www.medicinapaliativa.es

ORIGINAL

\title{
Sentido del sufrimiento en una muestra de mayores consagrados a la vida religiosa. Validación de la Escala Humanizar breve
}

\author{
José Carlos Bermejo, Marta Villacieros* y Hadil Hassoun \\ Centro San Camilo, Centro de Humanización de la Salud, Cantoblanco, Madrid, España
}

Recibido el 12 de abril de 2019

Aceptado el 17 de septiembre de 2019

\author{
PALABRAS CLAVE \\ Sentido del \\ sufrimiento, análisis \\ factorial confirmatorio, \\ Escala Humanizar, \\ cuidados paliativos, \\ religiosos consagrados.
}

\begin{abstract}
Resumen
Objetivo: Validación mediante análisis factorial confirmatorio de la Escala Humanizar breve en una muestra de adultos consagrados a la vida religiosa y comparación de las puntuaciones obtenidas por los religiosos con los datos publicados en población general.

Método: La Escala Humanizar breve consta de 14 ítems. Sus 2 factores recogen el sentido que se puede dar al sufrimiento como cambio (SS Cambio) y como carga (SS Carga). Con una muestra de $\mathrm{N}=190$ religiosos consagrados, se realizó un análisis factorial confirmatorio (estimación, mínimos cuadrados no ponderados sobre matriz de correlaciones policóricas), empleando los paquetes de R Lavaan y Semplot.

Resultados: Tras eliminar los ítems 1, 8 y 11, el análisis factorial confirmatorio confirmó el modelo de 2 factores, SS Cambio (5 ítems) y SS Carga (6 ítems). Los índices de ajuste obtenidos fueron adecuados: $\mathrm{RMSEA}=0,065 ; \mathrm{GFI}=0,992 ; \mathrm{SRMR}=0,079 ; \mathrm{CFI}=0,974 ; \mathrm{NNFI}=0,966 ; \mathrm{X2} / \mathrm{gl}=1,79$. Los pesos factoriales fueron significativos y mayores de 0,425. El alfa de Cronbach fue de 0,698 para el primer factor y de 0,712 para el segundo. La escala completa de 0,819 . Los factores mostraron una correlación de 0,658 ( $<<0,01)$. La media/ítem en la dimensión SS Carga fue mayor en población de religiosos consagrados (en este estudio; $M$ /ítem $=2,36$ ) que en cualquiera de los grupos de población general $(M /$ ítem < 1,85).

Conclusión: Se confirma la Escala Humanizar breve de 11 ítems para su uso en mayores religiosos, población en la que el sentido que se otorga al sufrimiento como carga es mayor que en el resto de la población. Se evidencia la importancia de valores y creencias, religiosidad y/o espiritualidad, a la hora de tratar el sufrimiento particularmente en cuidados paliativos.
\end{abstract}

\footnotetext{
${ }^{*}$ Autor para correspondencia:

Marta Villacieros

Centro de Humanización de la Salud, Sector Escultores 39, Tres Cantos, 28760 Madrid, España

Correo electrónico: investigacion@humanizar.es
}

DOI: 10.20986/medpal.2020.1091/2019

1134-248X/ ( 2020 Sociedad Española de Cuidados Paliativos. Publicado por Inspira Network. Todos los derechos reservados. 


\section{KEYWORDS}

Sense of suffering, confirmatory factor analysis, Humanizar scale, palliative care, religious life.

\begin{abstract}
Objective: Using confirmatory factor analysis, to validate the "Humanizar" scale, short version, in a sample of adults devoted to religious life, and to compare the scores in our sample with those reported for the general population.

Method: The "Humanizar" scale (14 items with a Likert scale) consists of 2 dimensions that give sense to suffering - as a change (SS Change) and as a burden (SS Burden). With $N=190$ subjects devoted to religious life, a confirmatory factor analysis (using unweighted least squares on a polychoric correlation matrix) was done using the R packages Lavaan and Semplot.

Results: After excluding items 1, 8, and 11, the confirmatory factor analysis confirmed the 2-dimensional model, SS Change (5 items) and SS Burden (6 items). The fitness indexes obtained were appropriate: $\mathrm{RMSEA}=0.065 ; \mathrm{GFI}=0.992 ; \mathrm{SRMR}=0.079 ; \mathrm{CFI}=0.974 ; \mathrm{NNFI}=0.966 ; \mathrm{X} 2 / \mathrm{df}=1.79$. The weight factor for all items was significant and greater than 0.425 . Cronbach's alpha was 0.698 for the first factor and 0.712 for the second one. For the full scale, 0.819 . Factors showed a correlation of $0.658(\mathrm{P}<0.01)$. Mean score/item for SS Burden was higher for the religious population (in this study; item $M=2.36$ ) than for any of the general population groups (item $M<1.85$ ).

Conclusion: The validity of the 11-item, short version of the "Humanizar" scale was confirmed for its use in a population devoted to religious life, a population where the meaning given to suffering as a burden is greater than in the general population. It shows the importance of values and beliefs, religion or spirituality, in dealing with suffering, particularly in palliative care.
\end{abstract}

Bermejo JC, Villacieros M, Hassoun $\mathrm{H}$. Sentido del sufrimiento en una muestra de mayores consagrados a la vida religiosa. Validación de la Escala Humanizar breve. Med Paliat. 2020;27(1):3-9

\section{INTRODUCCIÓN}

Según la Asociación Internacional para el Estudio del Dolor (IASP) ${ }^{1}$, se define el dolor como la sensación desagradable y la experiencia emocional asociadas con una lesión. El sufrimiento, sin embargo, se define como una respuesta de ansiedad, estrés o sensación de pérdida ${ }^{2}$ o como un estado afectivo cognitivo complejo caracterizado por la sensación de amenaza a la integridad de la persona y sentimientos de incapacidad para hacerle frente ${ }^{3-5}$. El sufrimiento no se debe confundir con el dolor, ya que el dolor o el miedo al dolor pueden generar sufrimiento. La sensación dolorosa está condicionada por otros factores, uno de ellos es la valoración personal del daño o el significado de la herida. Las personas que padecen dolor declaran que este se convierte en sufrimiento cuando su origen es desconocido, cuando no hay alivio o cuando su significado es funesto. Se convierte así en una de las realidades más conflictivas de la experiencia humana, experiencia que desafía nuestro sentido de búsqueda de paz y felicidad 6 .

Desde el ámbito de los cuidados paliativos, uno de los objetivos principales dentro del marco del cuidado holístico (que contempla, entre otros, el apoyo espiritual, el diálogo y la escucha activa ${ }^{7}$ ) es aliviar a las personas del sufrimiento, sea físico, emocional, psicológico o existencial. En esta línea, Cicely Saunders distinguió 2 tipos de dolor, el dolor físico y el dolor total o dolor psicológico y espiritual ante la muerte. Posicionó la atención al sufrimiento como objetivo central de los cuidados paliativos y reabrió la importancia de la dimensión espiritual ${ }^{8}$. Así, en su concepto de dolor total incluyó elementos sociales, emocionales y espirituales destacando que la experiencia total del paciente comprende tanto ansiedad, depresión y miedo como preocupación por la familia y la necesidad de encontrar un sentido a la situación?. Por ello, una de las perspectivas de trabajo se centra en comprender el origen y el sentido del sufrimiento, ya que desde las distintas tradiciones religiosas el sufrimiento tiene valor si se enfoca como oportunidad para la conexión espiritual y la trascendencia ${ }^{10}$.

El sentido que se le da al sufrimiento está muy condicionado por la edad, el momento del ciclo de vida o la etapa vital que se atraviesa, la educación recibida y las creencias o valores particulares de cada persona ${ }^{11-13}$. Muchos estudios se han centrado en encontrar la relación del sufrimiento con el bienestar espiritual ${ }^{14}$, tanto en población con enfermedad crónica ${ }^{15,16}$ como en cuidados paliativos ${ }^{17}$. También se ha incrementado la investigación sobre religión y espiritualidad, diferenciándola de creencia religiosa ${ }^{18,19}$. La espiritualidad es la capacidad humana de vincularse con valores, que abre la posibilidad autotrascendente, y el término religioso se refiere a la expresión de la propia espiritualidad ${ }^{6}$.

Las creencias religiosas embebidas en el sistema de creencias del propio individuo pueden influir de tal forma que lleguen a explicar algunas de sus estrategias de afrontamiento, así como determinados comportamientos ${ }^{20,21}$. El afrontamiento religioso se define, pues, como la búsqueda de apoyo espiritual depositando esperanza en creencias de consolación frente a lo inevitable o irreparable de las pérdidas sufridas ${ }^{22}$. 
En este sentido, la persona creyente cuenta con un recurso más ante el sufrimiento que genera la proximidad de la muerte. Según Gómez Sancho, es capaz de reconciliarse con la muerte y aceptarla ya que, para ella, no es el fin de su existencia individual ${ }^{6}$. Por ello, desde el ámbito de los cuidados paliativos se han desarrollado una gran variedad de instrumentos de medida de religiosidad y espiritualidad ${ }^{23-27}$.

El sufrimiento es inevitablemente humano y humanamente inevitable. Somos una combinación de materia y espíritu, independientemente de la interpretación que le se dé. Como acontecimiento humano, siempre presente, solo hay diferentes modos de enfrentarlo y nos plantea la cuestión del sentido 6 .

Desde el ámbito filosófico y antropológico, el sentido del sufrimiento ha dado lugar a mucha literatura. Max Scheler (filósofo y fenomenólogo) ${ }^{28}$ y Viktor Frankl (analista existencial y terapeuta ${ }^{29}$ abordan la problemática del sufrimiento, convergiendo ambos pensamientos en la posibilidad de hacer del sufrimiento un camino hacia el encuentro con el sentido de la vida. Frankl plantea que el ser humano se encuentra en constante búsqueda de sentido. Para él, la persona, además de ser homo sapiens, es homo patiens, un ser capaz de sufrir dignamente o de encontrar un sentido a sus sufrimientos y enfrentarlos humanamente ${ }^{29}$.

Scheler postula que para el ser humano lo importante es el perfeccionamiento de nuestra persona, entendiéndolo como su disminución para alcanzar su destino moral, independiente de la vida animal ${ }^{28}$. Es decir, que vivimos un estrato superior unido a lo espiritual independiente del animal. Y desde ahí el sufrimiento se entiende como sacrificio en el camino al ser humano total. Cuanta más aceptación del sufrimiento, más cerca del hombre existencial, metafísico y religioso, estaremos ${ }^{28}$.

Sin embargo, desde el ámbito de la investigación, las creencias sobre el sufrimiento y su sentido se han desatendido. Existen escalas que miden creencias sobre el sufrimiento, como la Views of Suffering Scale (VOSS), que valora un rango amplio de sistemas de creencias incluyendo las perspectivas teísticas, budistas, ateístas hindúes y no ortodoxas $^{11}$. Sin embargo, esta escala se desarrolló en Estados Unidos y en muestras de estudiantes con las limitaciones que suponen las diferencias culturales y el nivel de madurez y vivencias de una muestra en el rango de edad mencionado. Desarrollada en población general adulta (entre 18 y 78 años) en España, la Escala Humanizar breve $(\mathrm{EHb})^{30}$ evalúa el sentido que se le puede dar al sufrimiento. Esta escala incluye 2 grandes factores: el sentido del sufrimiento como cambio (SS Cambio) (utilidad que se le puede otorgar al sufrimiento como palanca o motor de cambio y oportunidad) y el sentido del sufrimiento como carga (SS Carga) (utilidad para sobrellevar o asumir ciertas cargas entendiéndolas como fortuitas, como sacrificio o como esfuerzo). El sentido que estas escalas dan al sufrimiento podría alinearse con los definidos por Frankl ${ }^{29}$ y Scheler ${ }^{28}$, respectivamente.

$\mathrm{La}$ escala $\mathrm{EHb}^{30}$ ha detectado diferencias en el sentido que se le da al sufrimiento en función de las creencias y la práctica de las personas. Los cristianos y los que practican oración o meditación otorgan más sentido al sufrimiento desde ambos puntos de vista, como cambio y como carga. Además, ambos factores muestran una asociación elevada, por lo que los autores concluyen que el sentido otorgado al sufrimiento se relaciona con la cultura religiosa, la judeo- cristiana (en este caso), que comprende que la carga, en caso de ser aceptada (o dotada de sentido), puede constituir crecimiento para la persona ${ }^{28,30}$.

Si esto es así, personas consagradas a la vida religiosa cristiana (sean miembros de institutos seculares, órdenes religiosas o pertenecientes al clero diocesano), cuyas creencias en este sentido se suponen más arraigadas, deberían de obtener puntuaciones más elevadas en la subescala de $\mathrm{EHb}$, SS Carga. Además, este resultado supondría un paso más en el proceso de validación de la escala, constatando el contenido de los ítems.

Por ello, esta investigación tuvo como objetivo, en primer lugar, la validación de la estructura del instrumento mediante análisis factorial confirmatorio en una muestra de adultos consagrados a la vida religiosa, y en segundo lugar, la comparación de las puntuaciones obtenidas en la escala por los religiosos con los datos publicados en población general ${ }^{30}$.

\section{PACIENTES Y MÉTODOS}

\section{Población de estudio}

Criterios de inclusión: la población diana fueron personas consagradas a la vida religiosa católica, todos ellos miembros de institutos seculares y órdenes religiosas ubicadas en distintas provincias de España. Criterios de exclusión: se excluyeron aquellos que padecían un deterioro cognitivo tal que impidiera la capacidad para comprender y contestar al cuestionario.

De una población de 290 religiosos sin deterioro cognitivo, aceptaron participar en el estudio 190 (tasa de respuesta del 65,5\%).

De los 190 participantes, el 94,7\% (180) eran mujeres y el $5,3 \%$ (10) hombres. Aun siendo una muestra mayoritaria de mujeres, se mantiene la muestra de hombres ya que también en la población de origen se da una situación similar (30 \% de religiosos hombres vs. $70 \%$ mujeres). La edad media fue de 84 años ( $\pm 7,14$ [64-98]); todos ellos son residentes en centros para religiosos mayores (enfermerías) asistidas por una misma fundación dedicada a la asistencia a religiosos en edad avanzada, desde hacía 4,04 años $( \pm 5,49)$ de media. De ellos, un $24 \%$ (45) tenían estudios de bachillerato o inferiores, un $59 \%$ (112) estudios de nivel medio (formación profesional/diplomatura/ técnico) y un $17 \%$ (33) licenciatura o estudios superiores.

\section{Instrumento}

$\mathrm{La} \mathrm{EHb}^{30}$ se compone de un total de 14 ítems con opciones de respuesta tipo Likert que van del 1 (Totalmente en desacuerdo) al 5 (Totalmente de acuerdo).

La escala incluye 2 factores, el SS Cambio (ítems 1, 4, 5, $7,8,10,11,13)$, que recoge la utilidad del sufrimiento para transformarse positivamente como palanca de cambio para salir de algunas situaciones de la vida o como fuente de energía para buscar lo que a uno le falta (ejemplo: ítem 5. El sufrimiento es un obstáculo a vencer y así lograr la paz interior), y el SS Carga (ítems 2, 3, 6, 9, 12, 14), que incluye el sentido que se le puede dar al sufrimiento cuando se afronta incluso ante algunas situaciones, que pudiendo ser evitadas no lo son, 
con cierto nivel de pasividad. Esta escala explica también la utilidad del sufrimiento cuando ocurre de manera fortuita, como si existiera una cuota de sufrimiento con la que alguien tiene que cargar, o como consecuencia de las malas acciones del ser humano (ejemplo: ítem 2. El ser humano debe pagar por sus actos irresponsables, por eso sufre).

Además de los 14 ítems de esta escala, en el cuestionario autoadministrado se incluyeron preguntas para recoger variables sociodemográficas, nivel de formación y tiempo residiendo en la enfermería.

\section{Procedimiento}

Los directores de un total de 24 residencias fueron contactados telefónicamente para explicarles los objetivos del estudio e invitarlos a participar en la investigación. Los enfermeros responsables en cada uno de los 21 centros que accedieron a participar fueron instruidos sobre cómo administrar los cuestionarios. Todos los participantes firmaron previamente un consentimiento informado y, con el fin de mantener la confidencialidad de los datos y el anonimato, los cuestionarios fueron codificados con un número. Los enfermeros responsables también fueron instruidos para ayudar a completar los cuestionarios a aquellas personas con limitaciones físicas (no cognitivas), sin influir en las respuestas del participante.

El estudio fue revisado por el Comité Ético Asistencial San Camilo y se llevó a cabo siguiendo los principios de la Declaración de Helsinki.

\section{Métodos estadísticos}

Para la realización de los análisis descriptivos se utilizó SPSSv20. Para el análisis factorial confirmatorio y la fiabilidad, el programa R Studio empleando los paquetes Lavaan ${ }^{31}$ y Semplot ${ }^{32}$.

Se llevó a cabo utilizando el método de estimación ULS (mínimos cuadrados no ponderados) sobre la matriz de correlaciones policóricas. Los indicadores de ajuste para valorar el modelo fueron el error cuadrático medio de aproximación (RMSEA $<0,08)$, análisis de residuos mediante el índice SRMR $(\leq 0,08)$, el índice de bondad de ajuste $(G F I>0,90)$, los índices de ajuste comparativos ( $\mathrm{CFI}>0,95$; NNFI > 0,95) y el valor de chi-cuadrado dividido por los grados de libertad $\left(X^{2} / \mathrm{gl}\right)$, ya que dicho estadístico es sensible al tamaño muestral. La fiabilidad de la escala se calculó mediante el índice de consistencia interna alfa de Cronbach.

\section{RESULTADOS}

\section{Análisis de ítems y fiabilidad}

Los ítems 1, 8 y 11 de la EHb publicada ${ }^{30}$, en esta muestra de religiosos consagrados, obtuvieron valores de correlación elemento con el total de la escala corregida $(\mathrm{Hc})$ inferiores a $0,3\left(\mathrm{Hc}_{1}=0,069 ; \mathrm{Hc}_{8}=0,288\right.$ y Hc $\left.\mathrm{H}_{11}=0,258\right)$ por lo que se eliminaron. Además, estos ítems obtuvieron las medias más elevadas y las menores varianzas. En la Tabla I se muestran

Tabla I. Análisis de los ítems de la Escala Humanizar breve para religiosos consagrados (alfa de Cronbach $=0,819$ )

\begin{tabular}{|c|c|c|c|c|c|c|c|}
\hline $\begin{array}{c}\text { Ítems de la EHb para religiosos consagrados, } \\
\text { nueva numeración (antigua) }\end{array}$ & Media & DT & Var & Mín & Máx & $\begin{array}{l}\text { Correlación } \\
\text { elemento-total } \\
\text { corregida }(\mathrm{Hc})\end{array}$ & $\begin{array}{c}\text { Alfa de Cronbach } \\
\text { si se elimina el } \\
\text { elemento }\end{array}$ \\
\hline $\begin{array}{l}1 \text { (2). El ser humano debe pagar por sus actos } \\
\text { irresponsables, por eso sufre }\end{array}$ & 2,37 & 1,344 & 1,805 & 1 & 5 & 0,402 & 0,813 \\
\hline $\begin{array}{l}2 \text { (3). El sufrimiento es consecuencia de las acciones } \\
\text { malas que se han cometido en la vida }\end{array}$ & 1,91 & 1,19 & 1,416 & 1 & 5 & 0,528 & 0,801 \\
\hline 3 (4). El sufrimiento es necesario para aprender & 3,48 & 1,29 & 1,664 & 1 & 5 & 0,352 & 0,817 \\
\hline $\begin{array}{l}4 \text { (5). El sufrimiento es un obstáculo a vencer y } \\
\text { así lograr la paz interior }\end{array}$ & 3,25 & 1,355 & 1,835 & 1 & 5 & 0,496 & 0,804 \\
\hline $\begin{array}{l}5(6) . \text { El origen del sufrimiento es el deseo, el } \\
\text { egoísmo }\end{array}$ & 2,64 & 1,301 & 1,693 & 1 & 5 & 0,466 & 0,807 \\
\hline $\begin{array}{l}6 \text { (7). El sufrimiento es una señal de que uno } \\
\text { debería cambiar algo }\end{array}$ & 3,12 & 1,218 & 1,483 & 1 & 5 & 0,546 & 0,799 \\
\hline $\begin{array}{l}7 \text { (9). El sufrimiento llega debido a la oposición o } \\
\text { alejamiento de lo Divino }\end{array}$ & 2,74 & 1,412 & 1,994 & 1 & 5 & 0,539 & 0,800 \\
\hline $\begin{array}{l}8 \text { (10). El sufrimiento se debe a la no aceptación } \\
\text { del dolor, a la lucha interna }\end{array}$ & 3,19 & 1,275 & 1,626 & 1 & 5 & 0,579 & 0,796 \\
\hline $\begin{array}{l}9 \text { (12). Lo provocan los ancestros, ofendidos por } \\
\text { los delitos cometidos o faltas morales }\end{array}$ & 1,89 & 1,07 & 1,145 & 1 & 5 & 0,448 & 0,809 \\
\hline $\begin{array}{l}10(13) . \text { El sufrimiento es una fuente de energía } \\
\text { para buscar lo que te falta }\end{array}$ & 3,2 & 1,248 & 1,557 & 1 & 5 & 0,505 & 0,803 \\
\hline $\begin{array}{l}11 \text { (14). Ya que existe una cantidad de sufrimiento, } \\
\text { si yo cojo mi parte al resto le queda menos }\end{array}$ & 2,64 & 1,411 & 1,99 & 1 & 5 & 0,499 & 0,804 \\
\hline
\end{tabular}


los estadísticos descriptivos de los ítems de la escala con su nueva numeración.

La fiabilidad del cuestionario obtenida mediante el coeficiente alfa de Cronbach fue adecuada, siendo 0,698 para el factor SS Cambio (ítems 3, 4, 6, 8 y 10 según la nueva numeración) y 0,712 para el factor SS Carga (ítems 1, 2, 5, 7, 9 y 11). La escala completa (11 ítems) obtuvo un alfa de 0,819.

\section{Análisis factorial confirmatorio}

Los índices de ajuste obtenidos en el análisis factorial confirmatorio de los 11 ítems en 2 factores fueron adecuados: $\mathrm{RMSEA}=0,065 ; \mathrm{GFI}=0,992 ;$ SRMR =0,079; $\mathrm{CFI}=0,974$; $\mathrm{NNFI}=0,966$. El índice Chi-cuadrado $(43)=77,23(p<0,05)$, por lo que $X^{2} / \mathrm{gl}=1,79$. En todos los casos las saturaciones de los ítems en los factores resultaron estadísticamente significativas $(p<0,001)$ y sustantivas (mayores a 0,425$)$ (Figura 1$)$. Respecto a la correlación entre ambos factores, esta fue significativa, moderada $(r=0,659 ; p<0,001)$ y mayor que la publicada en población general $(31 ; r=0,56 ; p<0,01)$.

La diferencia de medias (por ítem) entre las subescalas en este estudio (de 0,88 puntos) resultó estadísticamente significativa $(p<0,001)$, siendo mayor la puntuación media por ítem de la escala SS Cambio $(M=3,24)$ que la de SS Carga $(M=2,36)$. No se encontraron diferencias en las puntuaciones de la escala o subescalas de este estudio en función del sexo, nivel de estudios, edad o tiempo de residencia.

Por último, en la Tabla II se muestran los estadísticos descriptivos de la EHb para religiosos consagrados y las publicadas en el artículo original ${ }^{30}$. Para poder comparar puntuaciones entre ambas escalas (y sus subescalas) se calcularon las medias/ítem. Como se observa, la dimensión SS Cambio entre población creyente (consagrada o no) y practicante de oración y/o meditación obtiene medias/ítem equivalentes
(M/ítem $=3,2)$, mientras que la media/ítem en la dimensión SS Carga es mayor en población de religiosos consagrados (es decir, en este estudio; $M /$ ítem $=2,36$ ) que en cualquiera de los grupos de población general $(M /$ ítem $<1,85)$.

\section{DISCUSIÓN}

El objetivo de este estudio era la validación de la EHb en adultos consagrados a la vida religiosa, y la comparación de las puntuaciones obtenidas por los religiosos con los datos obtenidos en población general ${ }^{30}$. Los resultados confirman el modelo bidimensional publicado y el planteamiento de partida, en que se hipotetizaba que, teniendo la población de consagrados religiosos unas creencias más arraigadas en cuanto el SS Carga, obtendrían mayores puntuaciones en dicha subescala, confirmándose no solo la validez de estructura, sino también la validez de contenido de la escala.

Dados los índices de ajuste obtenidos en el AFC, similares a los conseguidos previamente en población general, se confirma la estructura de 2 factores: SS Cambio explica la utilidad del sufrimiento para transformarse positivamente y SS Carga recoge el sentido que se le puede dar al sufrimiento cuando se entiende como una carga a sobrellevar con cierto nivel de pasividad.

Los resultados de este estudio confirman la hipótesis planteada: entre población de adultos consagrados a la vida religiosa, el SS Carga obtiene mayores puntuaciones ${ }^{28}$. Además, ya que se mantiene el SS Cambio, la mayor asociación entre ambas dimensiones parece reducir el límite entre los 2 factores. Es decir, cuanta mayor religiosidad hay, más sentido se le da al sufrimiento, sea con propósito de cambio y oportunidad o como carga a sobrellevar. 0 , como concluyen los autores, las 2 subescalas están correlacionadas porque ambas dotan de un sentido útil al sufrimiento y precisamen-

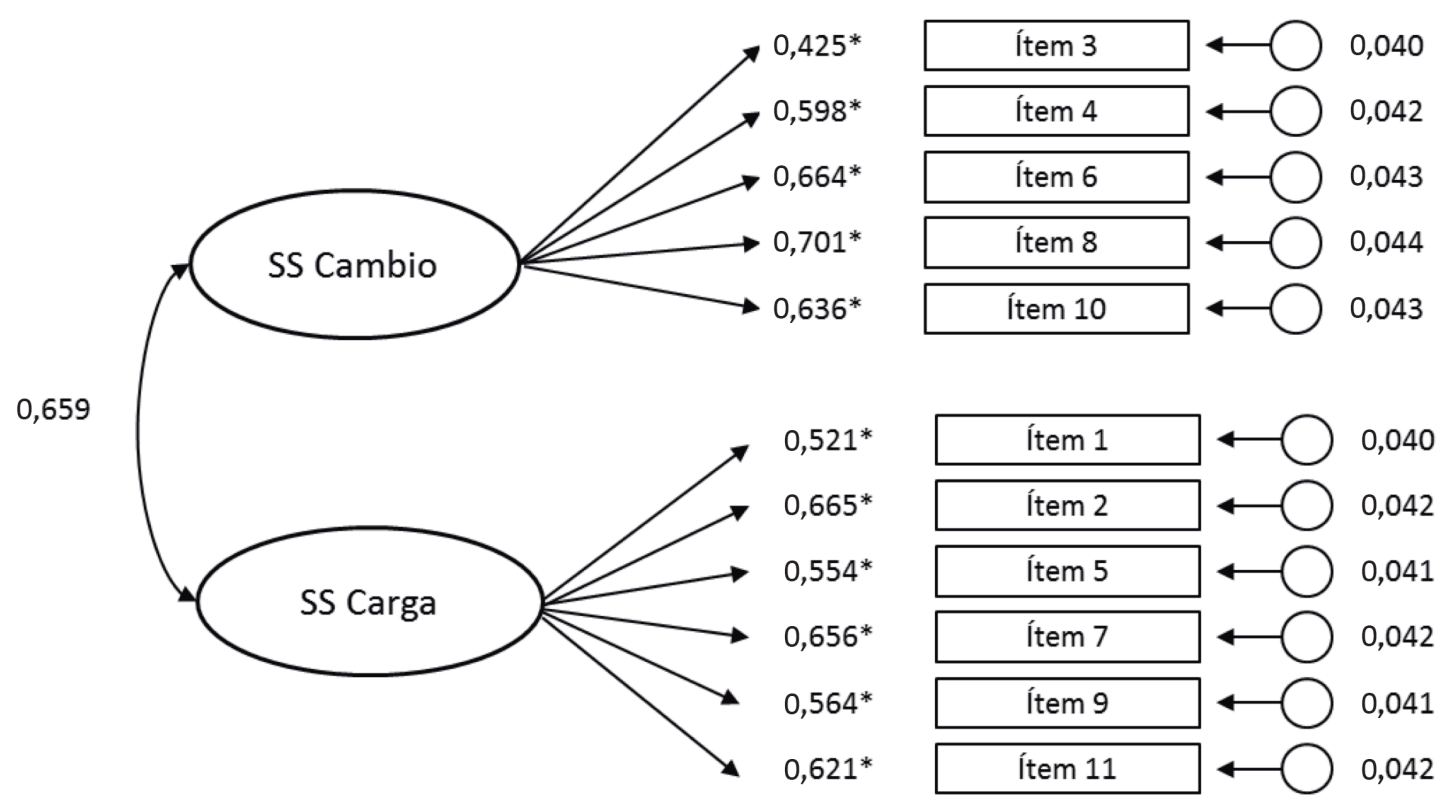

Figura 1. Modelo factorial de la Escala Humanizar breve para religiosos consagrados. Valores de los pesos estandarizados y de los errores de medida. SS Cambio: sentido del sufrimiento como cambio.

SS Carga: sentido del sufrimiento como carga. * $\mathrm{p}<0,001$. 
Tabla II. Estadísticos descriptivos de la EHb y sus subescalas en este estudio y en el artículo original ${ }^{30}$

\begin{tabular}{|c|c|c|c|c|c|c|c|c|c|c|}
\hline \multirow[b]{2}{*}{ Subescala } & \multicolumn{6}{|c|}{ Datos de este estudio } & \multicolumn{4}{|c|}{ Datos estudio original30 } \\
\hline & M & DT & Var & Mín & Máx & M/ítem & $\begin{array}{c}\text { M/ítem } \\
\text { ateos }\end{array}$ & $\begin{array}{l}\text { M/ítem } \\
\text { crist }\end{array}$ & $\begin{array}{c}\text { M/ítem orac/ } \\
\text { med No }\end{array}$ & $\begin{array}{c}\text { M/ítem } \\
\text { orac/med Sí }\end{array}$ \\
\hline SS Cambio & 16,24 & 4,22 & 17,88 & 5 & 25 & 3,24 & 2,94 & 3,20 & 2,88 & 3,24 \\
\hline SS Carga & 14,18 & 4,97 & 24,71 & 6 & 30 & 2,36 & 1,53 & 1,84 & 1,6 & 1,85 \\
\hline
\end{tabular}

DT: desviación típica. M: media. Máx: máximo. Mín: mínimo. SS: sentido del sufrimiento. SS Cambio: sentido del sufrimiento como cambio. SS Carga: sentido del sufrimiento como carga.

Nota: Las medias/ítem del estudio original se calculan dividiendo las medias publicadas entre el número de ítems de la subescala (8 en el caso de SS Cambio y 6 en el caso de SS Carga).

te son los cristianos, los que practican oración ${ }^{30}$ y ahora los religiosos consagrados, los que lo encuentran.

En esta población tan particular, que valora más que la población general la dimensión del SS Carga a sobrellevar, los ítems de la EHb 1, 8 y $11^{30}$ pierden fuerza. Estos ítems recogían un significado más romántico y menos sacrificado (ítem 1. Sufrir puede ser algo trascendental, puede llevar a una vida nueva; ítem 8 . El sufrimiento está íntimamente ligado al amor; e ítem 11. La experiencia de sufrimiento sirve para cambiar la manera de ver la vida), razón por la que se han eliminado.

Como se recoge en la literatura ${ }^{4,6,8,12,13,18,19,28-30,33}$ y se observa en los datos, el factor religiosidad está subyacente a la comprensión del sentido del sufrimiento, más presente si cabe entre población de mayores consagrados a la vida religiosa ${ }^{33}$. En el proceso de envejecimiento se presentan dificultades específicas, relativas a la salud física, mental, emocional, relacional y espiritual. Además, actualmente, los religiosos en Europa se enfrentan a preguntas que les ponen de frente a la soledad y la muerte, y a las que es difícil encontrar un sentido, como afrontar la posible desaparición de los institutos y sus obras (colegios, hospitales, residencias, centros de servicios sociales) ${ }^{33}$.

Sin contradecir el principio médico de prevención y alivio del sufrimiento, los resultados de este estudio evidencian que el significado y el valor del sufrimiento van más allá de la medicina y apoyan las perspectivas de los distintos credos, que sugieren que la medicina y la bioética tienen mucho que ganar de la consideración del discurso religioso sobre el sufrimiento ${ }^{11,34}$.

Como expone Gómez Sancho ${ }^{6}$, el sufrimiento en sí mismo no es algo deseable, sino irremediable y se presenta en muchas formas, esperadas o inesperadas. Lo que sí se puede modificar es la forma de enfrentarse a él. Darle sentido puede ser una de las claves para que el proceso de la persona que sufre se dirija hacia la amargura o hacia el crecimiento interior. Como decía V. Frankl, el hombre no se destruye por sufrir, sino por sufrir sin ningún sentido ${ }^{29}$. Así, encontrar sentido se considera una de las necesidades espirituales de final de vida 6 , no solo en población religiosa o creyente.

Tanto los mecanismos psicoemocionales y espirituales como los sistemas de creencias (religiosas o no) que se ponen en marcha ante el sufrimiento se pueden trabajar desde los equipos multidisciplinares de las UCP. La EHb y, ahora, su validación para población de religiosos consagrados, constituye una herramienta para un primer paso de detección.
Como limitaciones del estudio, señalar que la muestra mayoritaria de mujeres podría estar generando un sesgo de género. Para generar evidencia empírica que asegure que el constructo que se evalúa (sentido del sufrimiento) tiene el mismo significado en ambos grupos (hombres y mujeres), habría que continuar el proceso de validación de esta escala, realizando estudios de invarianza ${ }^{35,36}$ con muestras más equilibradas en cuanto a sexo. Otros pasos sucesivos serán de mayor rango de edad y procedentes de otras religiones.

Resumiendo, en este estudio se confirma la validez de la $\mathrm{EHb}$ de 11 ítems para su uso en población de mayores religiosos, donde el sentido que se otorga al sufrimiento como carga es mayor que en población general, y se evidencia la importancia de la consideración de los valores y creencias de las personas, su religiosidad y/o espiritualidad, a la hora de atender y tratar su sufrimiento en cualquier ámbito, destacando el de los cuidados paliativos, donde la situación de final de vida pone en jaque la calidad del proceso de afrontamiento.

\section{CONFLICTOS DE INTERESES}

Los autores declaramos que no presentamos ningún conflicto de intereses relevante en este artículo.

\section{FUENTES DE FINANCIACIÓN}

Investigación subvencionada por el Ministerio de Sanidad, Consumo y Bienestar Social para la realización de actividades de interés general con cargo a la asignación tributaria del Impuesto sobre la Renta de las Personas Físicas.

\section{BIBLIOGRAFÍA}

1. International Association for the Study of Pain (IASP). Classification of chronic pain. Second ed (revised). Seattle: IASP PRESS; 1994.

2. González F. ¿Es el dolor crónico un problema psiquiátrico aún no clasificado? La fibromialgia. Revista electrónica de psiquiatría. 1999;3:1-21.

3. Cassell EJ. The nature of suffering and the goals of medicine. N Engl J Med. 1982;306:639-45.

4. Bayés R. Psicología del sufrimiento y de la muerte. Anuario de Psicología. 1998;29:5-17. 
5. Chapman CR, Gavrin J. Suffering and its relationship to pain. J Palliat Care. 1993;9:5-13.

6. Gómez Sancho M. Dolor y sufrimiento. El problema del sentido. Rev Soc Esp Dolor. 1998;5:144-58.

7. Muñoz Devesa A, Morales Moreno I, Bermejo Higuera JC, Galán González Serna JM. La Enfermería y los cuidados del sufrimiento espiritual. Index Enferm. 2014;23:153-6.

8. Benito E, Grupo de Espiritualidad de la Sociedad Española de Cuidados Paliativos (SECPAL). Los cuidados espirituales, entraña de los cuidados paliativos. Med Paliat. 2011;18:1-3.

9. Clark D. Total pain, disciplinary power and the body in the work of Cicely Saunders, 1958-1967. Soc Sci Med. 1999;49:727-36.

10. Norris RS. The paradox of healing pain. Religion. 2009;39:22-33.

11. Hale-Smith A, Park CL, Edmondson D. Measuring beliefs about suffering: development of the views of suffering scale. Psychol Assess. 2012;24:855-66.

12. Bermejo JC. Sufrimiento y exclusión desde la fe. $1 .^{\mathrm{a}}$ ed. Santander: Sal Terrae; 2005.

13. Bermejo JC. El cristiano y la enfermedad. $4{ }^{\text {a }}$ ed. Barcelona: Editorial CPL; 2011.

14. Velásquez MP, Rebolledo D, González J. Cuidados de enfermería en el sufrimiento espiritual aplicando el modelo de Jane Watson. Salud, Arte, Cuidado. 2009;2:8-13.

15. Yunta ER. El sentido del sufrimiento: experiencia de los enfermos de cáncer. Ars Med. 2016;29:53-66.

16. Krikorian A. Valoración del sufrimiento en pacientes con cáncer avanzado. Psicooncología. 2008;5:257.

17. García-Campayo J, Sanz-Carrillo C, Lasa Labaca G. La enfermedad y el sentido del sufrimiento. Cuadernos de Bioética. 1996;25:50-7.

18. Lee $B Y$, Newberg $A B$. Religion and health: A review and critical analysis. Zygon. 2005;40:443-68.

19. Masters K, Hooker S. Religion, spirituality, and health. En: Paloutzian RF, Park CL, editores. Handbook of the psychology of religion and spirituality. $2^{\text {nd }}$ ed. New York, NY: Guilford Press; 2014.

20. Aldwin C. Stress, coping, and development: An integrative perspective. $2^{\text {nd }}$ ed. New York, NY: Guilford Press; 2007.

21. Bussing A, Ostermann T, Matthiessen PF. The role of religion and spirituality in medical patients in Germany. J Relig Health. 2005;44:321-40.

22. Krzemien D. Estilos de personalidad y afrontamiento situacional frente al envejecimiento en la mujer. Interam J Psychol. 2007;41:139-50.
23. Hearn J, Higginson IJ. Development and validation of a core outcome measure for palliative care: the Palliative Care Outcome Scale. Qual Saf Health Care. 1999;8:219-27.

24. Brady MJ, Peterman AH, Fitchett G, Cella D. The expanded version of the Functional Assessment of Chronic Illness Therapy spiritual well-being scale (FACIT-Sp-Ex): initial report of psychometric properties. Ann Behav Med. 1999;21:129.

25. Ironson G, Solomon GF, Balbin EG, O'Cleirigh C, George A, Kumar M, et al. The Ironson-woods Spirituality/Religiousness Index is associated with long survival, health behaviors, less distress and low cortisol in people with HIV/AIDS. Ann Behav Med. 2002;24:34-48.

26. The WHOQOL HIV Group. Initial steps to developing the World Health Organization's Quality of Life Instrument (WHOQOL) module for international assessment in HIV/AIDS. AIDS Care. 2003;15:347-57.

27. Sharma RK, Astrow AB, Texeira K, Sulmasy DP. The Spiritual Needs Assessment for Patients (SNAP): development and validation of a comprehensive instrument to assess unmet spiritual needs. J Pain Symptom Manage. 2012;44:44-51.

28. Scheler M. El puesto del hombre en el cosmos. Trad. de José Gaos. Biblioteca de Obras Maestras del Pensamiento. 1. ${ }^{a}$ ed. Buenos Aires: Losada; 2008.

29. Frankl VE. La voz que clama en demanda de sentido. En: Psicoterapia y humanismo ¿tiene sentido la vida? $\left(6 .{ }^{a}\right.$ reimp. de la

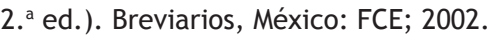

30. Villacieros M, Bermejo JC, Hassoun H. Validación psicométrica de la Escala Humanizar breve sobre el sentido del sufrimiento. J Healthc Qual Res. 2019;34:86-92.

31. Rosseel Y. Lavaan: An R package for structural equation modeling. J Stat Soft. 2012;48:1-36.

32. Fox J. Structural equation modeling with the SEM package in $\mathrm{R}$. Struct Equ Modeling. 2006;13:465-86.

33. Bermejo JC. Envejecimiento en la vida religiosa. $1 .^{\mathrm{a}}$ ed. Bilbao: Desclee de Brouwer; 2013.

34. Fitzpatrick SJ, Kerridge IH, Jordens CF, Zoloth L, Tollefsen C, Tsomo KL, et al. Religious perspectives on human suffering: Implications for Medicine and Bioethics. J Relig Health. 2015;55:159-73.

35. Byrne BM. Testing for multigroup equivalence of a measuring instrument: a walk through the process. Psicothema. 2008;2:872-82.

36. Van der Schoot R, Lugtig P, Hox J. A checklist for testing measurement invariance. Eur J Dev Psychol. 2012;9:486-92. 